\title{
Quantum mechanical calculation of ejected electron spectra for ion-atom collisions
}

\author{
Emil Y Sidky and C D Lin \\ James R Mcdonald Laboratory, Department of Physics, Kansas State University, Manhattan, KS \\ 66506-2604, USA
}

Received 27 January 1998

\begin{abstract}
We present a quantum mechanical method for calculating the ejected electron probability distribution in ion-atom collisions. The time-dependent Schrödinger equation, formulated with a classical straight-line trajectory for the internuclear motion, is integrated directly on a two-centre momentum space grid. The time-dependent wavefunction at large internuclear separation is analysed to extract the ejected momentum distribution as well as excitation and charge transfer amplitudes. Calculations have been performed at collision velocities of 1,2 and 5 au for proton and antiproton collisions with atomic hydrogen, at a few impact parameters. Excitation and charge transfer probabilities are also calculated to check against results obtained from the close-coupling method. The ejected electron momentum distributions are shown.
\end{abstract}

\section{Introduction}

Over the past two decades great strides have been made in the study of ion-atom collisions. Experimentally cross sections for the dominant processes for many collision systems have been measured. These measurements have stimulated much theoretical work. While various methods have been used from time to time to interpret experimental results, the closecoupling methods have been proven to be the workhorse for the prediction of experimental results over a broad range of energies and collision systems. The close-coupling method is based on expanding the time-dependent wavefunction in a finite configuration space volume and basis functions are designed to account for the two-centre nature of an ion-atom collision event. The finite-volume aspect of close coupling limits its application to excitation and charge transfer processes. By using pseudostates to approximate the continuum electrons in an averaged way, the close-coupling method has also been relatively successful in predicting total ionization cross sections. However, a complete measurement of the ionization also determines the momentum distribution of the ejected electron which is beyond the scope of the close-coupling method.

In recent years a novel experimental technique for atomic collisions is the cold target recoil ion momentum spectroscopy (COLTRIMS) (Ullrich et al 1997), which strives to determine fully the momentum components of the reaction products. For ionization events, this offers complete determination of all the momentum components of a three-body breakup process and offers a quantum mechanically complete experiment. Data for many collision systems from COLTRIMS experiments have been reported (Wood et al 1997, Moshammer et al 1997), but the theoretical interpretations available have been rather 
limited. In the regime where the ionization can be treated perturbatively, the planewave Born approximation and the continuum distorted-wave-eikonal initial-state (CDWEIS) theory have proven to be quite successful (Rodriguez et al 1995, Fainstein 1996, O'Rourke and Crothers 1997). Although close-coupling expansion based on atomic orbitals only on one centre can be formulated (Morishita et al 1996) to describe ionization in full detail, in principle the method is inadequate to account for the two-centre nature of the collision event and thus is limited to the higher energy region as well. The more interesting regime for ionization where little is understood is the low-energy region and collisions involving multiply charged ions. Different mechanisms for ionization at low energies have been addressed, in particular, the importance of the so-called saddle-point electrons and its region of prominence, have been widely debated (Olson 1983, Winter and Lin 1984, DuBois 1992, Irby et al 1993, Suárez et al 1993, Pieksma et al 1994).

To our knowledge, there are a few other attempts at describing ionization in the nonperturbative regime. The classical trajectory Monte Carlo (CTMC) method has been extensively performed to compare with COLTRIMS experiments with some success and some failure (Dörner et al 1996, Abdallah et al 1997). This method is limited by its classical treatment of the motion of the electron and its associated ambiguities. An attempt is also being made (Illescas et al 1997) to use CTMC to determine roughly the ejected electron spectra and then tailor a set of pseudostates for a close-coupling calculation. Direct integration of the time-dependent Schrödinger equation (Wells et al 1996) on a two-dimensional grid for protons on hydrogen, using the soft-core Coulomb potential, shows electron distributions as the projectile ion passes the target. This calculation gives a good qualitative representation of the collision, but cannot be directly compared with the COLTRIMS experiment owing to the model nature of the calculation. Another approach is the work of Macek and Ovchinnikov (Ovchinnikov et al 1997). They perform calculations by a Sturmian expansion in a Fourier space. With some approximations, they have made predictions on the ejected electron spectra for ionization of atomic hydrogen by protons at low energies.

In this paper we set out to calculate the full electron momentum distribution of the ejected electron for ion-atom collisions. Our aim is to be able to address a wide range of collision systems as well as collision energies. Thus in the higher energy region we hope to reproduce results obtainable from perturbation calculations. In the low-energy region we also hope to obtain a full description of the ionization process, and thus to evaluate the importance of the saddle-point electrons, at different collision velocities and for various systems, in comparison with the so-called direct ionization and electron capture to the continuum. It is our goal that we obtain the full electron momentum spectra in a single calculation - the theoretical equivalent of a COLTRIMS experiment. In fact, we aim to solve the full time-dependent Schrödinger equation exactly, in the numerical sense, which automatically also gives excitation and charge transfer probabilities. Since it is a 'complete' calculation, it is expected to describe both the dominant and the weak processes. In fact at low collision energies and for collisions with multiply charged ions, ionization is a weak process. To ensure that our calculation is meaningful we need to be able to obtain accurate excitation and charge transfer probabilities as well. This has the advantage that results from the close-coupling calculation can be used to check the new method. By allowing the method to be applicable to the higher velocity region, we can also compare with the results obtained from perturbation theory.

We envision that this approach will give full information on all the channels in an ion-atom collision event. Any form of close-coupling calculation is not desirable since this requires a large basis set and the evaluation of many two-centre matrix elements. It is 
more convenient to solve the time-dependent Schrödinger equation directly in configuration space (Schultz et al 1997) or in momentum space (Momberger et al 1996), where there is no need to evaluate the large number of matrix elements present in the close-coupling method. Both methods consist of a lattice discretization in their respective spaces. In order to achieve reasonable representation of the oscillating time-dependent wavefunction, the number of grid points in each dimension cannot be too limited. Thus in a realistic threedimensional calculation the memory requirement for a typical lattice calculation is quite large and can be performed only on massively parallel machines. In addition the momentum space calculation is computationally intensive due to the presence of convolution integrals. Granted both large-scale calculations were originally intended for relativistic collisions, total cross sections for the nonrelativistic case have been reported, which can be obtained by the close-coupling method with less computational effort.

Our method also directly integrates the time-dependent Schrödinger equation. We construct the electron wavefunction in momentum space. The wavefunction is expanded in spherical harmonics about both target and projectile centres, both of which are stationary in momentum space, assuming a straight-line projectile trajectory. The two-centre expansion limits the number of harmonics necessary thereby reducing the computational effort. Indeed, our program runs on a PC Pentium Pro instead of a multiprocessor machine. The main advantage to expressing the wavefunction in momentum space is that one knows the wavefunction is confined, i.e. the wavefunction goes to zero for large momentum. To avoid difficulties in evaluating the convolution integrals present in the momentum space Schrödinger equation, the basis is transformed to coordinate space; a similar strategy to the three-dimensional Fourier grid method (Brau and Semay 1998).

To our knowledge we obtain the first quantum mechanical ejected electron distributions in the nonperturbative regime for nonzero impact parameter. In this paper we introduce the two-centre momentum space discretization (TCMSD) method, and show results to argue for the method's validity. The first section explains the general strategy for efficiently calculating the time derivative of the electron wavefunction. The second section discusses the construction of the wavefunction for ion-atom collisions, how to propagate it, and how to analyse the final state. The third section shows results for proton and antiproton impact on hydrogen, focusing mainly on the ionization process. Finally, future directions for the TCMSD method are discussed.

\section{Preliminary comments}

The program developed here solves the time-dependent Schrödinger equation,

$$
\mathrm{i} \frac{\partial \psi}{\partial t}=H \psi
$$

One can expand the electronic wavefunction in some arbitrary, time-independent finite basis,

$$
\psi(\vec{r}, t)=\sum_{k=1}^{M} c_{k}(t) \Xi_{k}(\vec{r}),
$$

where $k$ labels each basis state and all the time dependence is taken into the coefficients $c_{k}$. If $\psi$ is completely represented by the basis $\Xi$ for all time $t$, then the coefficients $c_{k}$ are determined by evaluating the wavefunction at $M$ points! More specifically one finds the time derivative of the coefficients:

$$
\frac{\partial c_{k}(t)}{\partial t}=\sum_{j=1}^{M}\left(M^{-1}\right)_{k j} d_{j}, \quad d_{j}=\frac{\partial \psi\left(\vec{r}_{j}, t\right)}{\partial t}, \quad M_{j k}=\Xi_{k}\left(\vec{r}_{j}\right) .
$$


Equation (3) follows from equation (2) with time-independent basis functions. The Schrödinger equation provides us with the vector $d_{j}$, which is the time derivative of the wavefunction at the points $r_{j}$. The matrix $M_{j k}$ is simply the basis functions evaluated at the points $r_{j}$. Equation (3) is adequate when the expansion in equation (2) is truly complete. If this is not the case, then it is wise to overdetermine the system by specifying more points $r_{j}$ than basis functions $\Xi_{k}$. The time derivative of the basis coefficients is arrived at by a linear least-squares fit based on values at the points $r_{j}$. The matrix $M_{j k}$ is the so-called design matrix for the linear fit, and is in general rectangular. Methods of solving this system, even for our complex-valued case, can be found in Press et al (1992).

\section{The two-centre momentum space discretization method for ion-atom collisions}

We confine our scope to true one-electron collisions, where we allow arbitrary nuclear charges on both centres. Generalization to spherically symmetric model potentials, for treating quasi-one-electron systems, is straightforward. The collision system is viewed in the standard semiclassical framework, where the internuclear motion is classical and the electron is treated fully quantum mechanically. Furthermore, the projectile motion is assumed rectilinear with constant velocity $\vec{v}$ and impact parameter $\vec{b}$. Thus the resulting Schrödinger equation views the electron moving in a time-dependent potential, arising from both the target and projectile Coulomb attraction:

$\mathrm{i} \frac{\partial \psi(\vec{r}, t)}{\partial t}=\left(-\frac{1}{2} \nabla^{2}-\frac{Z_{T}}{|\vec{r}|}-\frac{Z_{P}}{|\vec{r}-\vec{R}|}\right) \psi(\vec{r}, t), \quad \vec{R}=\vec{v} t+\vec{b}$.

The coordinate system which we use here is the natural frame, where the projectile velocity $\vec{v}$ is along the $x$-axis, the impact parameter $\vec{b}$ is along the $y$-axis and the $z$-axis is perpendicular to the collision plane. Atomic units are used throughout.

\subsection{Wavefunction expansion}

We introduce the wavefunction expansion in momentum space. To account for the twocentre nature of the collision system the momentum space wavefunction $\Phi$ is expanded in spherical harmonics about both nuclear centres:

$\Phi(\vec{p}, t)=\sum_{l, m} \tilde{T}_{l, m}(p, t) Y_{l, m}(\hat{p})+\mathrm{e}^{-\mathrm{i}\left(\vec{p} \cdot \vec{R}-\frac{1}{2} v^{2} t\right)} \sum_{l, m} \tilde{P}_{l, m}(q, t) Y_{l, m}(\hat{q}), \quad \vec{q}=\vec{p}-\vec{v}$.

$\tilde{T}$ and $\tilde{P}$ are radial functions in momentum space which depend on time and the magnitude of the momentum with respect to the target and projectile respectively. The phase factor in front of the projectile centred expansion is the momentum space version of the electron translation factor (ETF) which results from the separation between the nuclear centres in configuration space. One can expect that a limited number of harmonics is necessary to represent the wavefunction about each centre, since the two-centre nature is accounted for and the ETF is included explicitly. One can employ $B$-splines to represent the radial functions up to a finite momentum $p_{f}$ after which an asymptotic form accounts for the large momentum tail. For this work we limit ourselves to the second-order $B$-splines, the so-called 'hat' functions (labelled $\tilde{B}_{i}$ here):

$$
\tilde{T}_{l, m}(p, t)=\sum_{i=1}^{N-1} c_{i}^{l m}(t) \tilde{B}_{i}(p) .
$$


Isolating the target expansion the asymptotic piece is:

$$
\Phi(\vec{p}, t)=\sum_{l, m} \frac{c_{N}^{l m}(t)}{p^{4+l}} Y_{l, m}(\hat{p}) \quad \text { for } p>p_{f} .
$$

To simplify the notation, we label the asymptotic radial term $1 / p^{4+l}$ by $\tilde{B}_{N}(p)$ even though it is not a $B$-spline. A similar expansion in terms of the coefficients $d_{i}^{l m}$ applies to the projectile centre. The large momentum components of the wavefunction separate neatly into target and projectile parts, since large momentum occurs only when the electron is near one or the other nucleus.

\subsection{Propagation}

Elaborating on the idea discussed in section 2 we describe the method for obtaining the timedependent coefficients $c_{i}^{l m}(t)$ and $d_{i}^{l m}(t)$. First, the configuration space representations of the basis functions, defined by equations (5)-(7), are obtained by the inverse Fourier transform. Second, the formulae for the time derivative of $c_{i}^{l m}(t)$ and $d_{i}^{l m}(t)$ are derived, in configuration space. Finally, the coefficients are propagated by a standard Runge-Kutta method with fixed step size. After propagation is completed the total electronic wavefunction, in either configuration or momentum space, is available for analysis.

Though it is convenient to express the electron wavefunction in momentum space for ion-atom collisions, it is undesirable to integrate the momentum space version of the Schrödinger equation due to the convolution integral for the potential energy term. Transforming equation (5) to configuration space avoids that difficulty. With the basis defined by equations (5)-(7) the inverse Fourier transform is obtained without much numerical effort. The inverse transform of equation (5) is largely analytic:

$\psi(\vec{r}, t)=\sum_{l, m} T_{l, m}(r, t) Y_{l, m}(\hat{r})+\mathrm{e}^{\mathrm{i}\left(\vec{v} \cdot \vec{r}-\frac{1}{2} v^{2} t\right)} \sum_{l, m} P_{l, m}(s, t) Y_{l, m}(\hat{s}), \quad \vec{s}=\vec{r}-\vec{R}$.

The inverse transform for the the radial functions is derived from the standard plane-wave expansion in spherical harmonics:

$$
T_{l, m}(r, t)=\mathrm{i}^{l} \sqrt{\frac{2}{\pi}} \int_{0}^{\infty} j_{l}(p r) \tilde{T}_{l, m}(p, t) p^{2} \mathrm{~d} p
$$

where $j_{l}$ is the spherical Bessel function. Likewise, the inverse transforms of the momentum space $B$-splines are denoted by $B_{i}$. With the linear $B$-spline representation the integral in equation (9) is analytic. The kinetic energy term for the basis is computed by inserting an additional $p^{2} / 2$ weighting factor into the integral of equation (9), and is denoted by ${ }^{\mathrm{KE}} B_{i}$. Substituting the wavefunction from equation (8) into equation (4) leads to the linear system of equations needed to propagate the time-dependent coefficients $c_{i}^{l m}(t)$ and $d_{i}^{l m}(t)$.

The time-dependent Schrödinger equation after inserting equation (8) becomes

$$
\begin{aligned}
\mathrm{i} \sum_{i, l, m} \frac{\partial c_{i}^{l m}(t)}{\partial t} B_{i}(r) Y_{l m}(\hat{r})+\mathrm{i} \mathrm{e}^{\mathrm{i}\left(\vec{v} \cdot \vec{r}-\frac{1}{2} v^{2} t\right)} \sum_{i, l, m} \frac{\partial d_{i}^{l m}(t)}{\partial t} B_{i}(s) Y_{l m}(\hat{s})=H \psi(\vec{r}, t) \\
H \psi(\vec{r}, t)=\sum_{i, l, m} c_{i}^{l m}(t)\left({ }^{\mathrm{KE}} B_{i}(r)-\frac{Z_{T}}{r} B_{i}(r)-\frac{Z_{P}}{s} B_{i}(r)\right) Y_{l m}(\hat{r}) \\
+\mathrm{e}^{\mathrm{i}\left(\vec{v} \cdot \vec{r}-\frac{1}{2} v^{2} t\right)} \sum_{i, l, m} d_{i}^{l m}(t)\left({ }^{\mathrm{KE}} B_{i}(s)-\frac{Z_{T}}{r} B_{i}(s)-\frac{Z_{P}}{s} B_{i}(s)\right) Y_{l m}(\hat{s}) .
\end{aligned}
$$


$H$ is the electron Hamiltonian shown on the right-hand side of equation (4). The time derivative on the left-hand side of the Schrödinger equation produces extra terms, since $s$ is a function of time, but they cancel with terms stemming from the ETF.

Standard close coupling proceeds by projecting equation (10) on the basis functions to isolate the time derivatives on the left-hand side. Instead we solve for the time derivatives of $c_{i}^{l m}$ and $d_{i}^{l m}$ by evaluating equation (10) at enough grid points to uniquely determine the time derivative of the basis coefficients. A set of points $r_{j}\left(s_{j}\right)$, stationary relative to the target (projectile) centre, are chosen. A specific example of how the points $r_{j}$ and $s_{j}$ are selected is given in section 4. Evaluation of equation (10) at the selected points yields the following linear system:

$$
\begin{aligned}
& A_{J K} x_{K}=b_{J} \\
& A_{J K}=\mathrm{i}\left(\begin{array}{cc}
B_{i}\left(\left|r_{j}\right|\right) Y_{l m}\left(\hat{r_{j}}\right) & \mathrm{e}^{\mathrm{i}\left(\vec{v} \cdot \vec{r}_{j}-\frac{1}{2} v^{2} t\right)} B_{i}\left(\left|r_{j}^{-}\right|\right) Y_{l m}\left(\hat{r_{j}^{-}}\right) \\
B_{i}\left(\left|s_{j}^{+}\right|\right) Y_{l m}\left(\hat{s_{j}^{+}}\right) & \mathrm{e}^{\mathrm{i}\left(\vec{v} \cdot\left(\overrightarrow{s_{j}}+\vec{R}\right)-\frac{1}{2} v^{2} t\right)} B_{i}\left(\left|s_{j}\right|\right) Y_{l m}\left(\hat{s_{j}}\right)
\end{array}\right) ; \\
& r_{j}^{-}=r_{j}-R, \quad s_{j}^{+}=s_{j}+R \\
& x_{K}=\left(\begin{array}{c}
\dot{c}_{i}^{l m} \\
\dot{d}_{i}^{l m}
\end{array}\right) ; \quad b_{J}=\left(\begin{array}{c}
H \psi\left(\vec{r}_{j}, t\right) \\
H \psi\left(\vec{s}_{j}+\vec{R}, t\right)
\end{array}\right) .
\end{aligned}
$$

$K$ is the index running through the target basis functions (which is the number of targetcentred harmonics times $N$ the number of target radial $B$-splines) and the projectile basis functions. $J$ is the total number of points $s_{j}$ and $r_{j}$. Thus equation (11) is set up as a linear least-squares fitting system, where the best values $x_{K}$ are sought based on the data $b_{K}$.

The procedure for a calculation starts by finding suitable coefficients, $c_{i}^{l m}\left(t_{0}\right)$, that represent the target state; $d_{i}^{l m}\left(t_{0}\right)$ are usually set to zero. Given the initial data the time derivatives are found through equation (11). A multitude of integration techniques are available to update the basis coefficients in time. We employ the straightforward, fourthorder Runge-Kutta with fixed step size for which an algorithm can be found in Press et al (1992). After propagation to some large time after the collision, the resulting momentum space wavefunction is analysed for bound-state amplitudes and ejected electron distributions.

\subsection{Extracting scattering amplitudes}

To analyse the proton-hydrogen collision system for bound states on either the target or projectile centre, we propagate the electron wavefunction until the two-centre interaction becomes negligible. Since the wavefunction, equation (5), is already separated into target and projectile parts, one need only project the radial functions, $\tilde{T}_{l, m}$ and $\tilde{P}_{l, m}$, onto the hydrogen bound-state radial functions:

$$
a_{n l m}^{T(P)}=\int_{0}^{\infty} F_{n l}(p) \tilde{T}(\tilde{P})_{l m}(p) p^{2} \mathrm{~d} p
$$

$F_{n l}$ is the hydrogenic radial function in momentum space. The validity of equation (12) rests upon the assumption that there is no significant overlap between the target and projectile centres. This is easily checked by finding $a_{n l m}^{T(P)}$ as a function of the internuclear separation when the projectile is receding. If the bound-state coefficients do not vary in magnitude, then the separation assumption is valid. This procedure does not guarantee that the phase of $a_{n l m}^{T(P)}$ will not undergo further evolution. However, for the purposes of calculating bound-state probabilities this procedure is sufficient.

To obtain the ejected electron spectrum, we proceed with the bound-state projection until the resulting coefficients are negligible. From the bound-state coefficients we build 
the complete bound component of the wavefunction, which is subsequently subtracted from the total wavefunction. For low-energy electrons one must take care that the bound-state component is really separated out. The momentum distribution of Rydberg states goes as $Z / n$, thus the lowest momentum, for which the ejected electron spectrum is reliable, is inversely proportional to the highest $n$-state that was projected out of the total wavefunction. As with the bound-state probabilities the momentum distribution of the ejected electron is checked for invariance with time after the collision.

\section{Test calculations and results}

We test TCMSD for selected impact parameters and projectile energies. Focusing on the proton-hydrogen system, capture and excitation probabilites are calculated for velocities 0.2 , 1 and 2 au. These probabilites are checked against close-coupling calculations. Turning our attention to ionization, we examine proton and antiproton impact on hydrogen for projectile velocities 1, 2 and 5 au. Since there is no other theory to check the ejected electron spectra, we select limiting cases where we can argue for their validity based on general principles for ionization in ion-atom collisions.

The same set of computational parameters is used for all calculations presented here with the exception that a two-centre basis is used for proton impact while a one-centre basis is used for antiproton impact. Spherical harmonics through $l=2$ are included to represent the angular part of the wavefunction about each centre. Due to reflection symmetry in the collision plane we keep only the six even harmonics out of nine. The corresponding radial functions in momentum space are discretized by $15 B$-splines at an equally spaced momentum interval of 0.1 au. Thus the asymptotic radial momentum dependence begins at $p_{f}=1.5 \mathrm{au}$. The total number of basis functions around each centre is $15 \times 6$, leading to 180 (90) basis functions for proton (antiproton) impact.

An overdetermined set of configuration space points is chosen to perform the leastsquares fitting. For the angular distribution six evenly distributed $(\pi / 3 \mathrm{rad}$ apart) azimuthal angles $\phi_{j}$ are selected, and likewise the inclination $\theta_{j}$ is selected at an interval of $\pi / 5$ but only three values are necessary. In the natural frame, where the $z$-axis is perpendicular to the collision plane, the wavefunction is the same for $\theta \rightarrow-\theta$, since the initial state is even. Thus only $\theta_{j} \leqslant \pi / 2$ are necessary. In the radial direction 30 points at a spacing of $\pi / p_{f}$ were chosen. The total number of fitting points for proton (antiproton) impact is 780 (390).

With these parameters the integration along the projectile trajectory starts at $x=-20$ au and ends at $x=20$ au. The trajectory is subdivided into 400 segments over which the Runge-Kutta integration is performed. Since the integration step is defined as a constant distance along the projectile path, the timesteps depend on the projectile velocity. Higher velocity corresponds to smaller timesteps, therefore a more accurate time integration. A rigid check on the stability of the time integration is the unitarity. For the calculations presented here the total integrated probability density varied half of a per cent from unity.

\subsection{Tests involving capture and excitation}

We choose three sets of collision parameters to test the program. First, we select a highvelocity projectile, $v=2$ au and $b=2.5 \mathrm{au}$, which is an easier test for TCMSD, since capture is not large and the interaction time is short. Second, we bring the velocity down to the 'matching' velocity, $v=1$ au and $b=2.5$ au, which is a more difficult test. Finally, we select a much lower projectile velocity $v=0.2$ au and $b=5.0$ au. Table 1 shows the 
Table 1. Comparison of TCMSD (ms) and close coupling (cc) bound-state probabilities for three tests. Test number 1 is for $v=2$ au and $b=2.5$ au, test number 2 is for $v=1$ au and $b=2.5$ au and test number 3 is for $v=0.2$ au and $b=5.0$ au. The labels el, ex and ca refer to elastic, excitation and capture respectively.

\begin{tabular}{cllllllll}
\hline Test number & 1s el & 2s ex & 2p -1 ex & 2p 1 ex & 1s ca & 2s ca & $2 \mathrm{p}-1 \mathrm{ca}$ & $2 \mathrm{p} 1 \mathrm{ca}$ \\
\hline $1 \mathrm{~ms}$ & 0.93 & 0.0034 & 0.027 & 0.0001 & 0.002 & 0.0003 & 0.0003 & 0.0 \\
$\mathrm{cc}$ & 0.94 & 0.0041 & 0.036 & 0.0006 & 0.003 & 0.0010 & 0.0002 & 0.0 \\
$2 \mathrm{~ms}$ & 0.74 & 0.0043 & 0.015 & 0.0005 & 0.13 & 0.023 & 0.0026 & 0.0034 \\
$\mathrm{cc}$ & 0.76 & 0.0049 & 0.007 & 0.0012 & 0.18 & 0.028 & 0.0011 & 0.0024 \\
$3 \mathrm{~ms}$ & 0.61 & 0.0 & 0.0 & 0.0 & 0.40 & 0.0 & 0.0 & 0.0 \\
$\mathrm{cc}$ & 0.54 & 0.0 & 0.0 & 0.0 & 0.46 & 0.0 & 0.0 & 0.0 \\
\hline
\end{tabular}

state-to-state probability comparison. The close-coupling calculation was carried out with all states up to $n=4$ on both centres.

Overall the agreement is acceptable. It must be borne in mind that computing the boundstate probabilities is a stringent test for TCMSD, since the radial functions are represented only by second-order $B$-splines. Thus differences of a few per cent are expected. The close-coupling calculation was performed without any continuum states, which are implicit in TCMSD. For the third test at $v=0.2$ au the collision can be modelled with just two states, the ground state on both centres. It is interesting that TCMSD also finds only two states, the ground state on either centre, with nonzero amplitude, yet disagrees with the close-coupling value by $10 \%$. Further investigation reveals that this discrepancy stems from inadequate treatment of polarization during the collision. Refining the low-momentum mesh to 0.04 au spacing improves the agreement (the elastic channel decreases to 0.57 and capture to the hydrogen $1 \mathrm{~s}$ increases to 0.43 ).

\subsection{Ionization}

We next turn to the ionization process, where we examine the ejected electron momentum distribution from proton and antiproton impact. Figure 1 $(a)$ shows the ejected electron distribution in the collision plane for $v=5$ au and $b=2$ au from proton impact, and likewise figure $1(b)$ shows the same for antiproton impact. For such a fast collision the projectile interaction is accurately approximated by an electric dipole pulse in the $y$-direction. Thus one expects that the electrons are emitted along the $y$-axis in both directions. Since the Born approximation is expected to be valid for this velocity, there should be no difference between proton and antiproton impact (the Born amplitude is proportional to the projectile charge, thus the sign of the charge is irrelevant upon squaring the amplitude to obtain the probability). The similarity between proton and antiproton impact at high velocity is apparent from comparing figures $1(a)$ and $(b)$. The total ionization probability calculated by TCMSD is 0.0135 and 0.0136 for proton and antiproton impact respectively.

Figure 2 shows the ejected electron momentum distribution at $b=2.5$ au for the lower velocity $v=2$ au. Comparing to figure 1 , we note that for proton collisions the electron density migrates to the region between the two centres in momentum space. This is an illustration of the 'two-centre' effect where the ionized electron is influenced by the attractive force from the projectile after the collision. This effect is often interpreted as a post-collision effect. The same post-collision effect can also explain the larger distribution of the electrons on the negative $p_{y}$ side for antiproton impact. The repulsive force between the electron and the antiproton favours electrons to move in the opposite direction. There is 


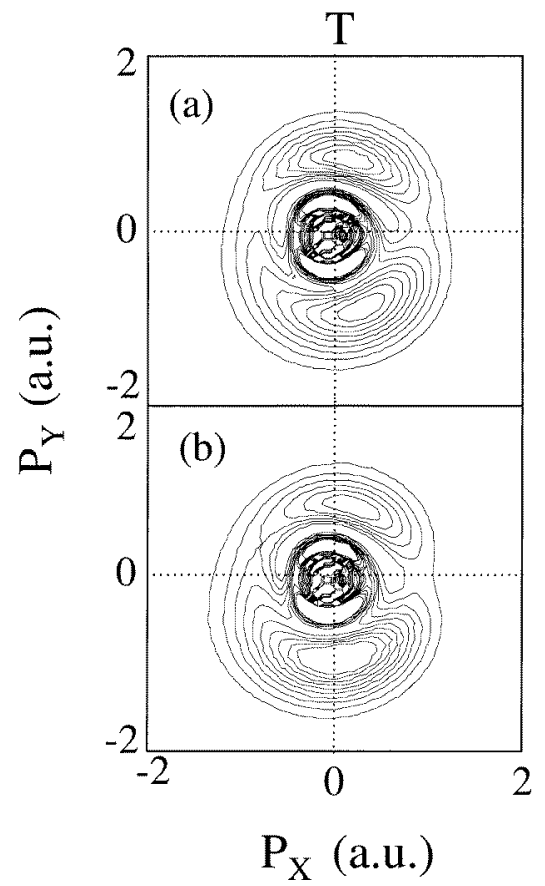

Figure 1. Ejected electron distribution for $(a)$ proton and $(b)$ antiproton impact on hydrogen for projectile speed 5 au and impact parameter $2 \mathrm{au}$. The vertical broken line, labelled $\mathrm{T}$, indicates the target longitudinal velocity.

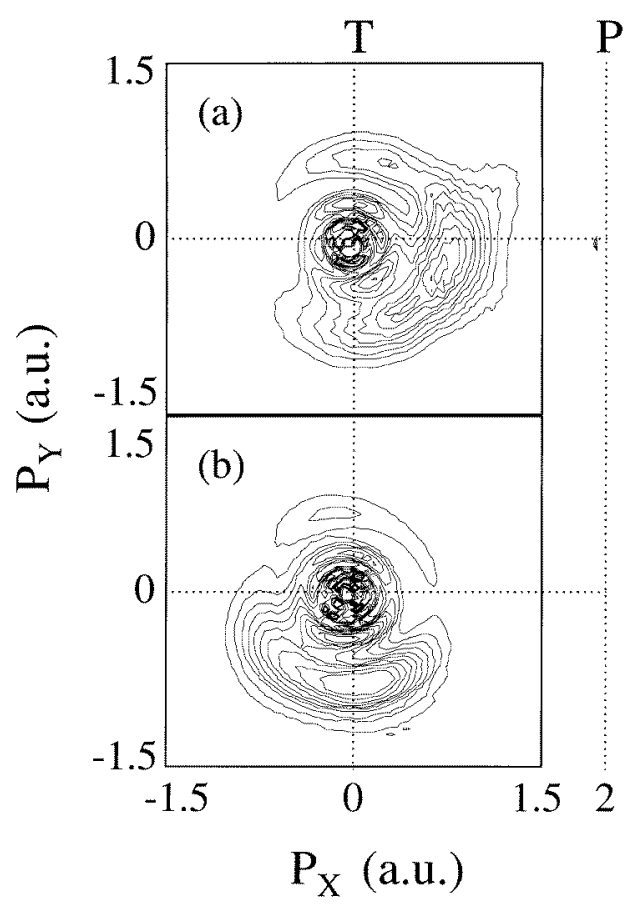

Figure 2. Ejected electron distribution for $(a)$ proton and (b) antiproton impact on hydrogen for projectile speed 2 au and impact parameter 2.5 au. The vertical broken line, labelled $\mathrm{T}(\mathrm{P})$, indicates the target (projectile) longitudinal velocity.

also a slight preference of the ejected electron to move toward the negative $x$-direction. For this collision the total ionization probability is 0.0457 for antiproton impact, and 0.0385 for proton impact, of which 0.0075 is attributed to electrons moving with the projectile centre. 


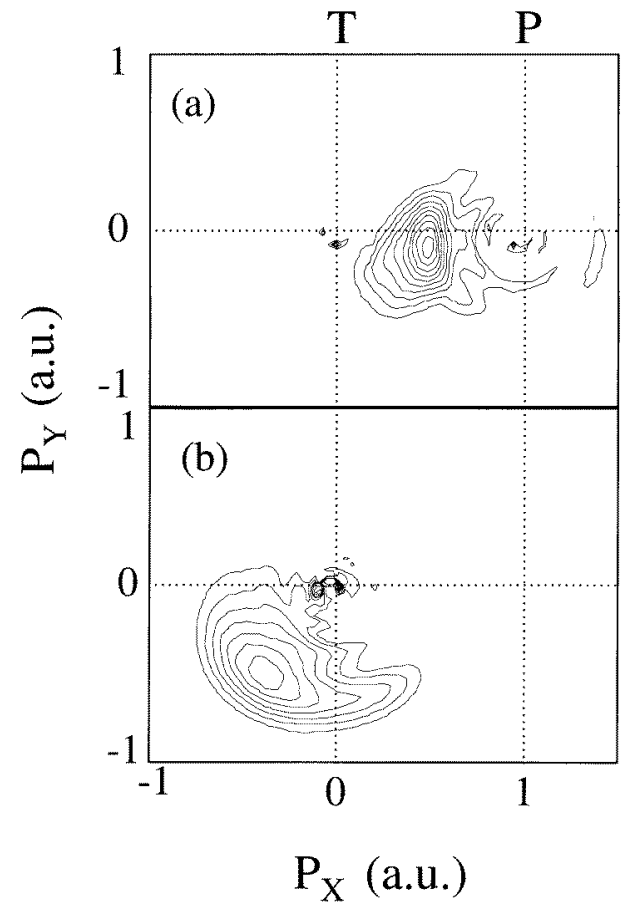

Figure 3. Ejected electron distribution for $(a)$ proton and (b) antiproton impact on hydrogen for projectile speed 1 au and impact parameter 2.5 au. The vertical broken line, labelled $\mathrm{T}(\mathrm{P})$, indicates the target (projectile) longitudinal velocity.

Figure 3 shows the ejected electron momentum distribution at $b=2.5$ au for collision velocity $v=1 \mathrm{au}$. For proton impact, the electron momentum is localized mostly between the two centres. From the two-centre calculation this means that the two centres contribute comparably to ionization, resulting in a momentum distribution for the ejected electrons that peaks near $v / 2$, where $v$ is the collision velocity. Although the location of the momentum peak coincides with the velocity of the saddle point of the potential surface in the coordinate space, the mechanism for this peak is not clear. At velocity matching the ejected electron is not easily influenced by the weak saddle of the potential surface. In figure $3(b)$ the momentum distribution by antiproton impact is peaked at negative $p_{y}$ as before in figure $2(b)$, but the shift is larger, reflecting the more important role of postcollision interaction for slower antiprotons. For this collision the total ionization probability is 0.0629 for antiproton impact, and 0.0401 for proton impact, of which 0.0295 is attributed to electrons moving with the projectile centre.

In figure 4 we plot the real part of the wavefunction in figure $3(a)$. The surface in figure 4 has many more oscillations than in figure 3(a). These oscillations arise from the large phase gradient represented by the ETF, which is incorporated analytically in TCMSD method. Interestingly, the plot in figure 4 cannot remain constant in time, since the ETF in equation (5) is time dependent. Yet, the probability distribution, figure 3(a), is stationary. Without the explicit ETF the numerical representation of the rapidly oscillating wavefunction becomes much more difficult.

\section{Summary and future directions}

We have presented a method for solving the time-dependent Schrödinger equation for ionatom collisions. Expressing the electron wavefunction in momentum space allows for 


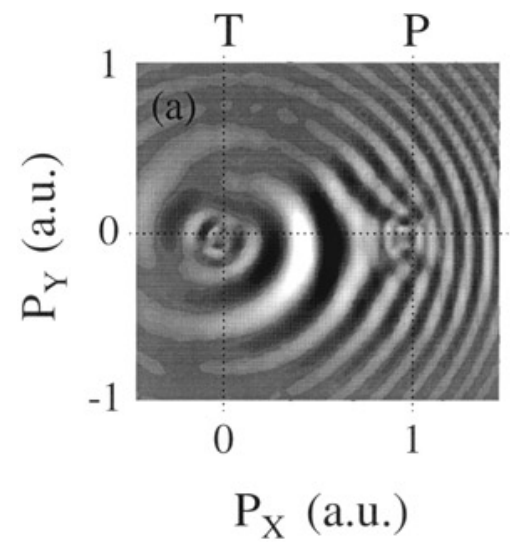

Figure 4. The real part of the wavefunction for the ejected electron for proton impact on hydrogen at $v=1$ au and $b=2.5$ au (same as figure $3(a)$ ). White, black and grey represent positive, negative and zero values respectively.

accurate representation of its positive energy component. The time derivative of the basis coefficients are found efficiently by a linear least-squares fit at selected points surrounding both target and projectile, avoiding a time-consuming calculation of complicated matrix elements. Probabilites for capture and excitation are computed by projection of projectile and target radial functions onto bound-state radial functions. The distribution of ejected electrons is formed by subtracting the bound component from the total wavefunction. Thus we obtain, for the first time, the full quantum mechanical ejected electron distributions for any impact parameter.

This paper, however, only reports tests for the TCMSD method; there are still improvements needed in order to calculate ionization distributions that can compare directly with experiment. The main improvement necessary is to adopt an uneven mesh, that can represent the low-momentum part of the wavefunction more accurately without wasting effort on representing the high-momentum part. Going to higher order $B$-splines would also reduce the number of splines necessary to represent the momentum space radial functions. The time integration should be generalized to a variable step size method and should allow for one-centre computation when the internuclear separation is large enough. These improvements together with model potentials, for nonhydrogenic atoms, will allow us to calculate the ejected electron momentum distribution for direct comparison with experiment in the near future.

\section{Acknowledgments}

We are grateful for helpful discussions with M Abdallah and T Morishita. This work is supported by the Division of Chemical Sciences, Office of Basic Energy Sciences, Office of Energy Research, US Department of Energy.

\section{References}

Abdallah M, Kravis S, Cocke C L, Wang Y, Rodriguez V D and Stöckli M 1997 Phys. Rev. A 56 2000-4

Brau F and Semay C 1998 J. Comput. Phys. 139 127-36

Dörner R, Khemliche H, Prior M H, Cocke C L, Gary J A, Olson R E, Mergel V, Ullrich J and Schmidt-Böcking H 1996 Phys. Rev. Lett. 77 4520-3

DuBois R D 1993 Phys. Rev. A 48 1123-8

Fainstein P D 1996 J. Phys. B: At. Mol. Opt. Phys. 29 L763-7

Illescas C, Rabadán I and Riera A 1997 J. Phys. B: At. Mol. Opt. Phys. 30 1765-84 
Irby V D, Datz S, Dittner P F, Jones N L, Krause H F and Vane C R 1993 Phys. Rev. A 47 2957-63

Momberger K, Belkacem A and Sørensen A H 1996 Phys. Rev. A 56 1605-22

Morishita T, Hino K, Watanabe S and Matsuzawa M 1996 Phys. Rev. A 53 2345-58

Moshammer R, Ullrich J, Kollmus H, Schmitt W, Unverzagt M, Schmidt-Böcking H, Wood C J and Olson R E 1997 Phys. Rev. A 56 1351-63

Olson R E 1983 Phys. Rev. A 271871

O'Rourke S F C and Crothers D S F 1997 J. Phys. B: At. Mol. Opt. Phys. 30 2443-54

Ovchinnikov S Y, Macek J H and Khrebtukov D B 1997 Phys. Rev. A 56 2872-86

Pieksma M, Ovchinnikov S Y, van Eck J, Westerveld W B and Niehaus A 1994 Phys. Rev. Lett. 73 46-9

Press W H, Teukolsky S A, Vetterling W T and Flannery B P 1992 Numerical Recipes in FORTRAN the Art of Scientific Computing 2nd edn (Cambridge: Cambridge University Press) pp 665, 704

Rodriguez V D, Wang Y D and Lin C D 1995 J. Phys. B: At. Mol. Opt. Phys. 28 L471-6

Schultz D R, Wells J C, Krstić P S and Reinhold C O 1997 Phys. Rev. A 56 3710-13

Suárez S, Garibotti C, Bernardi G, Focke P and Meckbach W 1993 Phys. Rev. A 48 4339-49

Ullrich J, Moshammer R, Dörner R, Jagutzki O, Mergel V, Schmidt-Böcking H and Spielberger L 1997 J. Phys. B: At. Mol. Opt. Phys. 30 2917-74

Wells J C, Schultz D R, Gavras P, and Pindzola M S 1996 Phys. Rev. A 54 593-604

Winter T G and Lin C D 1984 Phys. Rev. A 293071

Wood C J, Olson R E, Schmitt W, Moshammer R and Ullrich J 1997 Phys. Rev. A 56 3746-52 